\title{
Einführung in die Ölhydraulik
}

Von Dr.-Ing. Hans Jürgen Matthies

o. Professor em. an der

Technischen Universität Braunschweig

2., überarbeitete und erweiterte Auflage

Mit 275 Bildern und 17 Tafeln 
Prof. Dr.-Ing. Hans Jürgen Matthies

Geboren 1921 in Teterow in Mecklenburg. Von 1940 bis 1945 Kriegsdienst. Von 1946 bis 1949 Studium des Maschinenbaus an der Technischen Universität Stuttgart. Von 1950 bis 1954 wissenschaftlicher Assistent am Institut für Landmaschinen der Technischen Hochschule Braunschweig (Prof. Dr.-Ing. G. Segler), 1954 Promotion. Von 1954 bis 1958 Mitarbeiter der Firma Gebrüder Welger, Maschinenfabrik, Wolfenbüttel (Chefkonstrukteur). Von 1958 bis 1990 o. Professor für Landmaschinen an der Technischen Universität Braunschweig. Seit 1990 emeritiert. Seit 1974 o. Mitglied der Braunschweigischen Wissenschaftlichen Gesellschaft.

CIP-Titelaufnahme der Deutschen Bibliothek

Matthies, Hans J.:

Einführung in die Ölhydraulik / von H. J. Matthies.

2., überarb. u. erw. Aufl.

Stuttgart : Teubner, 1991

(Teubner-Studienbücher : Maschinenbau)

ISBN 978-3-519-16318-3 ISBN 978-3-322-94107-7 (eBook)

DOI 10.1007/978-3-322-94107-7

Das Werk einschließlich aller seiner Teile ist urheberrechtlich geschützt. Jede Verwertung außerhalb der engen Grenzen des Urheberrechtsgesetzes ist ohne Zustimmung des Verlages unzulässig und strafbar. Das gilt besonders für Vervielfältigungen, U'bersetzungen, Mikroverfilmungen und die Einspeicherung und Verarbeitung in elektronischen Systemen.

(c) B. G. Teubner Stuttgart 1991

Satz: Elsner \& Behrens GmbH, Oftersheim

Umschlaggestaltung: W. Koch, Sindelfingen 


\section{Vorwort}

Die Olhydraulik hat dem Maschinenbaukonstrukteur völlig neue Möglichkeiten für die Gestaltung seiner Maschinen und für die Verwirklichung neuer Konstruktionsideen eröffnet. Viele Funktionen, die früher mit mechanischen Mitteln nur unter erheblichem Aufwand erfüllt werden konnten, lassen sich mit Hilfe der Hydraulik einfacher und präziser verwirklichen. Darüber hinaus bietet die Ölhydraulik auch dem Anwender aufgrund der besseren und leichteren Bedienbarkeit der Maschinen und ihrer meist größeren Vielseitigkeit erhebliche Vorteile.

Der Maschinenbaustudent, nicht zuletzt aber auch der bereits in der Praxis tätige Ingenieur, hat oft Schwierigkeiten, sich einen Uberblick über den Stand der Technik auf dem Gebiet der Ölhydraulik zu verschaffen. Es fehlen ihm häufig die ausreichenden Grundkenntnisse, um die Funktion hydraulischer Geräte und Anlagen zu verstehen und um solche Anlagen für den eigenen Bereich selbst zu entwickeln.

Dieses Lehrbuch soll dazu beitragen, hier Abhilfe zu schaffen. Es wendet sich daher in erster Linie an den Maschinenbaustudenten und auch den in der Praxis tätigen Ingenieur, der sich die notwendigen Grundkenntnisse auf diesem Gebiete aneignen und sie bei der Arbeit in seinem Tätigkeitsbereich anwenden will.

Das Buch wurde aus einer vom Verfasser seit 1970 an der Technischen Universität Braunschweig gehaltenen Vorlesung entwickelt. Bei seiner Gestaltung wurde besonderer Wert auf didaktische Gesichtspunkte gelegt. Ein straff gegliederter Inhalt, die ausführliche Darstellung der für die Anwendung der Ölhydraulik notwendigen Grundlagen und die auf das wesentliche beschränkte, auf Systematik bedachte zeichnerische Darstellung der Geräte und Schaltungen erlauben es dem technisch ausgebildeten Leser, sich ohne Vorkenntnisse auf diesem Gebiet in die Ölhydraulik einzuarbeiten.

In der vorliegenden 2. Auflage wurden die Anwendungsbeispiele um einen neuen Abschnitt über sekundärgeregelte hydrostatische Antriebe und um weitere Beispiele aus den Gebieten der mobilen und der stationären Arbeitsmaschinen erweitert. Darüber hinaus wurden zahlreiche Verbesserungen vorgenommen. Für Hinweise und Anregungen dazu danke ich vielen Lesern; gleichzeitig bitte ich aber um Verständnis, wenn nicht alle Vorschläge berücksichtigt werden konnten.

Zum Aufbau der Vorlesung und zur Gestaltung der 1. Auflage des Buches haben die Herren Dr.-Ing. O. Böinghoff, D. Hoffmann, M. Kahrs und B. Link beigetragen. Dafür gilt ihnen mein Dank. Für die Mithilfe bei den Korrekturen und Ergänzungen zur 2. Auflage danke ich besonders den Herren Dipl.-Ing. H. Esders, Dr.-Ing. W. Friedrichsen und Dipl.-Ing. J. Möller. 


\section{Inhalt}

\section{Einführung}

1.1 Begriffe . . . . . . . . . . . . . . . . . . . . . . 11

1.2 Aufbau und Funktion ölhydraulischer Antriebe . . . . . . . . 12

1.3 Technische Eigenschaften ölhydraulischer Antriebe . . . . . . . 15

1.4 Geschichtliche Entwicklung der Ölhydraulik . . . . . . . . . . 16

2 Grundlagen für Entwicklung und Betrieb ölhydraulischer Antriebe

2.1 Grundlagen über Druckflüssigkeiten . . . . . . . . . . . . 20

2.1.1 Aufgaben und Anforderungen . . . . . . . . . . . . 20

2.1.2 Arten und Stoffdaten . . . . . . . . . . . . . . . 21

2.1.3 Physikalisches Verhalten . . . . . . . . . . . . . . 24

2.1.3.1 Viskositätsverhalten. 2.1.3.2 Dichteverhalten.

2.1.3.3 Luftaufnahmevermögen

2.2 Grundlagen aus der Hydrostatik . . . . . . . . . . . . . . . 31

2.2.1 Hydrostatisches Verhalten von Flüssigkeiten . . . . . . . 31

2.2.2 Energiewandlung mit Kolben und Zylinder . . . . . . . . 32

2.2.3 Energiewandlung mit rotierendem Verdränger . . . . . . . 33

2.3 Grundlagen aus der Hydrodynamik . . . . . . . . . . . . . 34

2.3.1 Kontinuitätsgleichung. . . . . . . . . . . . . . . 34

2.3.2 Bernoulli'sche Bewegungsgleichung . . . . . . . . . . 35

2.3.3 Druckverlust in Rohrleitungen . . . . . . . . . . . . 36

2.3.3.1 Grundlegende Betrachtungen. 2.3.3.2 Laminare Rohrströmung. 2.3.3.3 Turbulente Rohrströmung

2.3.4 Druckverlust in Krümmern und Leitungselementen . . . . . 43

2.3.5 Druckverlust in Ventilen und Geräten . . . . . . . . . 46

2.3.6 Volumenstrom durch Drosseln . . . . . . . . . . . . 46

2.3.7 Leckölverlust durch Spalte . . . . . . . . . . . . . 48

2.3.8 Kraftwirkung strömender Flüssigkeiten . . . . . . . . . 49

2.4 Grundlagen aus der Gleitlagertechnik . . . . . . . . . . . . 50

2.5 Schaltzeichen . . . . . . . . . . . . . . . . . . . . 53

3 Energiewandler für stetige Bewegung (Hydropumpen und -motoren)

3.1 Axialkolbenmaschinen . . . . . . . . . . . . . . . . . 59

3.1.1 Schrägachsenmaschinen . . . . . . . . . . . . . . 59

3.1.2 Schrägscheibenmaschinen . . . . . . . . . . . . . 62

3.1.3 Taumelscheibenmaschinen . . . . . . . . . . . . . 64

3.1.4 Berechnung von Axialkolbenmaschinen . . . . . . . . . 65 
3.2 Radialkolbenmaschinen . . . . . . . . . . . . . . . . 68

3.2.1 Innenbeaufschlagte Maschinen . . . . . . . . . . . . 68

3.2.2 Außenbeaufschlagte Maschinen. . . . . . . . . . . . 70

3.2.3 Berechnung von Radialkolbenmaschinen . . . . . . . . 70

3.3 Zahnrad- und Zahnringmaschinen . . . . . . . . . . . . . 71

3.3.1 Außenzahnradmaschinen. . . . . . . . . . . . . . 71

3.3.2 Innenzahnradmaschinen . . . . . . . . . . . . . . 73

3.3.3 Zahnringmaschinen . . . . . . . . . . . . . . . 74

3.3.4 Berechnung von Zahnrad- und Zahnringmaschinen . . . . . 75

3.4 Flügelzellenmaschinen . . . . . . . . . . . . . . . . . 75

3.4.1 Einhubige Maschinen . . . . . . . . . . . . . . . 76

3.4.2 Mehrhubige Maschinen . . . . . . . . . . . . . . 76

3.4.3 Berechnung von Flügelzellenmaschinen . . . . . . . . . 77

3.5 Sperr- und Rollflügelmaschinen . . . . . . . . . . . . . . 77

3.5.1 Sperrflügelmaschinen . . . . . . . . . . . . . . . 77

3.5.2 Rollflügelmaschinen . . . . . . . . . . . . . . . 78

3.5.3 Berechnung von Sperr- und Rollflügelmaschinen . . . . . . 78

3.6 Schraubenmaschinen . . . . . . . . . . . . . . . . . 79

3.7 Betriebsverhalten von Verdrängermaschinen . . . . . . . . . . 80

3.7.1 Betriebseigenschaften der Bauarten . . . . . . . . . . 80

3.7.2 Wirkungsgrade und Kennlinienfelder . . . . . . . . . . 80

3.7.3 Förderstrom- und Druckpulsation . . . . . . . . . . . 88

3.7.4 Pulsationsdämpfung . . . . . . . . . . . . . . . . 92

4 Energiewandler für absätzige Bewegung (Hydrozylinder, Schwenkmotoren)

4.1 Einfachwirkende Zylinder . . . . . . . . . . . . . . . . 96

4.1.1 Plunger- oder Tauchkolbenzylinder . . . . . . . . . . 96

4.1.2 Normaler einfachwirkender Zylinder . . . . . . . . . . . 96

4.1.3 Mehrfach- oder Teleskopzylinder . . . . . . . . . . . 97

4.2 Doppeltwirkende Zylinder . . . . . . . . . . . . . . . . . . . . . . . . 99

4.2.1 Zylinder mit zweiseitiger Kolbenstange (Gleichlaufzylinder) . . 99

4.2.2 Zylinder mit einseitiger Kolbenstange (Differentialzylinder) . . 100

4.3 Detailgestaltung und Einbau von Hydrozylindern . . . . . . . . . 101

4.3.1 Endlagendämpfung. . . . . . . . . . . . . . . . 101

4.3.2 Einbau von Hydrozylindern . . . . . . . . . . . . . 102

4.4 Schwenkmotoren. . . . . . . . . . . . . . . . . . . 102

4.4.1 Schwenkmotoren mit mechanischer Ubersetzung . . . . . . 103

4.4.2 Schwenkmotoren mit direkter Beaufschlagung. . . . . . . 104

\section{Elemente und Geräte zur Energiesteuerung und -regelung (Ventile)}

5.1 Betätigungsmittel für Ventile . . . . . . . . . . . . . . 105

5.1.1 Ubersicht . . . . . . . . . . . . . . . . . . . 105

5.1.2 Schaltende elektromechanische Wandler . . . . . . . . . 107 
5.1.3 Proportionalwirkende elektromechanische Wandler .

5.1.3.1 Geschichtliche Entwicklung. 5.1.3.2 Torque-Motoren.

5.1.3.3 Tauchspulen. 5.1.3.4 Proportionalmagnete

5.2 Wegeventile.

5.2.1 Konstruktive Gestaltung . . . . . . . . . . . . . . 114

5.2.2 Nichtdrosselnde Wegeventile . . . . . . . . . . . . 118

5.2.2.1 Direkt betätigte nichtdrosselnde Wegeventile. 5.2.2.2

Uber Vorsteuerventil betätigte nichtdrosselnde Wegeventile

5.2.3 Drosselnde Wegeventile . . . . . . . . . . .
5.2.3.1 Mechanisch betätigte drosselnde Wegeventile.
5.2 .3 .2

Elektromechanisch betätigte proportionalwirkende Wegeventile

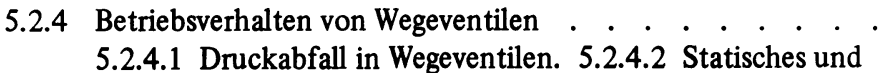
dynamisches Verhalten von proportionalwirkenden Wegeventilen

5.3 Sperrventile

5.3.1 Einfache Rückschlagventile . . . . . . . . . . . . . 132

5.3.2 Entsperrbare Rückschlagventile . . . . . . . . . . . 132

5.3.3 Drosselrückschlagventile . . . . . . . . . . . . . 132

5.4 Druckventile . . . . . . . . . . . . . . . . . . . . 133

5.4.1 Druckbegrenzungsventile . . . . . . . . . . . . . . 134

5.4.2 Druckverhältnisventile (Druckstufenventile) . . . . . . . 135

5.4.3 Folgeventile (Zuschaltventile) . . . . . . . . . . . . 136

5.4.4 Druckregel- oder Druckreduzierventile (Druckminderventile) . . 137

5.4.5 Differenzdruckregelventile (Druckgefälleventile) . . . . . . 137

5.4.6 Verhältnisdruckregelventile (Druckverhältnisventile). . . . . 138

5.4.7 Kombinierte Druckventile . . . . . . . . . . . . . 138

5.4.8 Proportional-Druckventile . . . . . . . . . . . . . 140

5.4.9 Betriebsverhalten von Druckventilen . . . . . . . . . . 141

5.5 Stromventile . . . . . . . . . . . . . . . . . . . 143

5.5.1 Drosselventile . . . . . . . . . . . . . . . . . 143

5.5.2 Stromregelventile . . . . . . . . . . . . . . . . 144

5.5.3 Stromteilventile. . . . . . . . . . . . . . . . . 146

5.5.4 Proportional-Stromventile . . . . . . . . . . . . . 147

5.5.5 Betriebsverhalten von Stromventilen . . . . . . . . . . 148

5.6 2-Wege-Einbauventile . . . . . . . . . . . . . . . . . 149

5.7 Ventilanschluß- und Verknüpfungsarten . . . . . . . . . . . 154

\section{Elemente und Geräte zur Energieübertragung}

6.1 Verbindungselemente . . . . . . . . . . . . . . 156

6.1.1 Rohr- und Schlauchleitungen . . . . . . . . . . . 156

6.1.2 Rohr- und Schlauchverbindungen . . . . . . . . . . . 159

6.2 Dichtungen. . . . . . . . . . . . . . . . . . . . . 160

6.2.1 Statische Dichtungen . . . . . . . . . . . . . . . 160

6.2.2 Dynamische Dichtungen . . . . . . . . . . . . . . 161 
6.2.3 Betriebsverhalten von Dichtungen . . . . . . . . . . . 162

6.3 Ölbehälter . . . . . . . . . . . . . . . . . . . . . 163

6.3.1 Allgemeines . . . . . . . . . . . . . . . . . . 163

6.3.2 Offene Ölbehälter . . . . . . . . . . . . . . . . . 163

6.3.3 Geschlossene Ölbehälter . . . . . . . . . . . . . . 164

6.4 Filter. . . . . . . . . . . . . . . . . . . . . . . 165

6.4.1 Allgemeines . . . . . . . . . . . . . . . . . . 165

6.4.2 Filterelemente . . . . . . . . . . . . . . . . . 166

6.4.3 Einsatzarten und Filterbauarten . . . . . . . . . . . 167

6.5 Hydrospeicher . . . . . . . . . . . . . . . . . . . 169

6.5.1 Allgemeines . . . . . . . . . . . . . . . . . . 169

6.5.2 Speicherbauarten . . . . . . . . . . . . . . . . 169

6.5.3 Berechnung von Speichern . . . . . . . . . . . . . 171

6.5.4 Sicherheitsbestimmungen . . . . . . . . . . . . . 173

6.6 Wärmetauscher . . . . . . . . . . . . . . . . . . . 174

6.6.1 Heizer (Vorwärmer) . . . . . . . . . . . . . . . 174

6.6 .2 Kühler . . . . . . . . . . . . . . . . . . . . 174

6.7 Schalt- und Meßgeräte . . . . . . . . . . . . . . . . . 176

7 Steuerung und Regelung hydrostatischer Antriebe

7.1 Allgemeines . . . . . . . . . . . . . . . . . . . . 178

7.2 Methoden zur Veränderung des Volumenstroms . . . . . . . . 179

7.2.1 Verwendung von Doppel-oder Mehrfachpumpen . . . . . . 180

7.2.2 Verwendung von Konstantpumpe und Drosselventil . . . . . 181

7.2.3 Verwendung von verstellbaren Verdrängermaschinen. . . . . 183

7.3 Steuerung mit Verstellpumpen . . . . . . . . . . . . . . 185

7.3.1 Grundlagen . . . . . . . . . . . . . . . . . . 185

7.3.2 Steuerungsarten. . . . . . . . . . . . . . . . . 186

7.3.2.1 Mechanische und elektrische Steuerungen. 7.3.2.2

Hydraulische Steuerungen. 7.3.2.3 Kombinierte Steuerungen

7.4 Regelung mit Verstellpumpen . . . . . . . . . . . . . . 192

7.4.1 Grundlagen . . . . . . . . . . . . . . . . . . 192

7.4.2 Regelungsarten . . . . . . . . . . . . . . . . . 193

7.4.2.1 Druckregelungen. 7.4.2.2 Stromregelungen. 7.4.2.3

Leistungsregelungen. 7.4.2.4 Kombinierte Regelungen

8 Planung und Betrieb hydrostatischer Anlagen

8.1 Schaltungsbeispiele . . . . . . . . . . . . . . . . . . 200

8.1.1 Schaltungen für einzelne Verbraucher . . . . . . . . . 201

8.1.2 Schaltungen für mehrere Verbraucher . . . . . . . . . 204

8.1.3 Systemschaltungen . . . . . . . . . . . . . . . . 207

8.2 Planung und Berechnung . . . . . . . . . . . . . . . . 211 
8.2.1 Bestimmung der Grunddaten . . . . . . . . . . . . 212

8.2.1.1 Planungsschritte. 8.2.1.2 Funktionsdiagramme.

8.2.1.3 Antriebsart und Betriebsdruck. 8.2.1.4 Planungsbeispiel

8.2.2 Leistungsfluß und Wirkungsgrade . . . . . . . . . . . 222

8.2.3 Wärmetechnische Auslegung. . . . . . . . . . . . . 223

8.2.3.1 Grundlagen. 8.2.3.2 Erwärmungsverlauf. 8.2.3.3 Berechnungsbeispiel

8.3 Uberlegungen zum Betriebsverhalten . . . . . . . . . . . . 229

8.3.1 Schwingungs- und Kavitationserscheinungen . . . . . . . 229

8.3.2 Eigenschaften der Druckflüssigkeit . . . . . . . . . . 230

8.3.3 Sonstige Einflüsse . . . . . . . . . . . . . . . . 231

\section{Anwendungsbeispiele}

9.1 Hydrostatische Getriebe . . . . . . . . . . . . . . . . 232

9.2 Sekundärgeregelte hydrostatische Antriebe . . . . . . . . . . 236

9.3 Hydraulik in mobilen Arbeitsmaschinen . . . . . . . . . . . 242

9.4 Hydraulik in stationären Arbeitsmaschinen . . . . . . . . . . 255

Normen und Richtlinien . . . . . . . . . . . . . . . . . 261

Literaturverzeichnis . . . . . . . . . . . . . . . . 263

Sachverzeichnis . . . . . . . . . . . . . . . . . . . 267 